\title{
Extragalactic gamma-ray background from AGN winds and star-forming galaxies in cosmological galaxy-formation models
}

\author{
A. Lamastra ${ }^{1,2}$, N. Menci ${ }^{1}$, F. Fiore ${ }^{1}$, L. A. Antonelli ${ }^{1,2}$, S. Colafrancesco ${ }^{3}$, D. Guetta ${ }^{1,4}$, and A. Stamerra ${ }^{5,6}$ \\ 1 INAF-Osservatorio Astronomico di Roma, via di Frascati 33, 00078 Monte Porzio Catone, Italy \\ e-mail: alessandra.lamastra@oa-roma.inaf.it \\ 2 SSDC-ASI, via del Politecnico, 00133 Roma, Italy \\ 3 School of Physics, University of the Witwatersrand, Private Bag 3, 2050 Johannesburg, South Africa \\ 4 Department of Physics and Optical Engineering, ORT Braude College, 21982 Karmiel, Israel \\ 5 INAF-Osservatorio Astrofisico di Torino, via Osservatorio, 20, 10025 Pino Torinese, Italy \\ ${ }^{6}$ Scuola Normale Superiore, Piazza dei Cavalieri 7, 56126 Pisa, Italy
}

Received 27 June 2017 / Accepted 10 September 2017

\begin{abstract}
We derive the contribution to the extragalactic gamma-ray background (EGB) from active galactic nuclei (AGN) winds and starforming galaxies by including a physical model for the $\gamma$-ray emission produced by relativistic protons accelerated by AGN-driven and supernova-driven shocks into a state-of-the-art semi-analytic model of galaxy formation. This is based on galaxy interactions as triggers of AGN accretion and starburst activity and on expanding blast waves as the mechanism to communicate outwards the energy injected into the interstellar medium by the active nucleus. We compare the model predictions with the latest measurement of the EGB spectrum performed by the Large Area Telescope (LAT) on board the Fermi Gamma-ray Space Telescope (Fermi) in the range between $100 \mathrm{MeV}$ and $820 \mathrm{GeV}$. We find that AGN winds can provide $\sim 35 \pm 15 \%$ of the observed EGB in the energy interval $E_{\gamma}=0.1-1 \mathrm{GeV}$, for $\sim 73 \pm 15 \%$ at $E_{\gamma}=1-10 \mathrm{GeV}$, and for $\sim 60 \pm 20 \%$ at $E_{\gamma} \gtrsim 10 \mathrm{GeV}$. The AGN wind contribution to the EGB is predicted to be larger by a factor of $\sim 3-5$ than that provided by star-forming galaxies (quiescent plus starburst) in the hierarchical clustering scenario. The cumulative $\gamma$-ray emission from AGN winds and blazars can account for the amplitude and spectral shape of the EGB, assuming the standard acceleration theory, and AGN wind parameters that agree with observations. We also compare the model prediction for the cumulative neutrino background from AGN winds with the most recent IceCube data. We find that for AGN winds with accelerated proton spectral index $p=2.2-2.3$, and taking into account internal absorption of $\gamma$-rays, the Fermi-LAT and IceCube data could be reproduced simultaneously.
\end{abstract}

Key words. galaxies: active - galaxies: evolution - galaxies: formation

\section{Introduction}

The extragalactic gamma-ray background (EGB) represents a superposition of all $\gamma$-ray sources, both individual and diffuse, from the Milky Way to the edge of the observable universe, and provides a view of the high-energy processes in the universe. Here we consider the total $\gamma$-ray photon flux produced outside of the Milky Way, including both resolved and unresolved sources. Indeed, the diffuse Galactic emission produced by the interaction of Galactic cosmic rays (CR), mainly protons and electrons, with the Galactic interstellar medium (ISM) and interstellar radiation field, is comparable to the EGB intensity and represents a strong foreground to the EGB measurement. The latter have recently been measured by the Large Area Telescope (LAT, Atwood et al. 2009 ) on board the Fermi Gamma-ray Space Telescope (Fermi), in the range between $100 \mathrm{Mev}$ and $820 \mathrm{GeV}$ (Ackermann et al. 2015). The EGB spectrum is well described by a power-law with exponential cut-off having a spectral index of $\sim 2.3$ and cut-off energy greater than $300 \mathrm{GeV}$.

How much different source classes contribute to the EGB remains one of the main unanswered questions of $\gamma$-ray astrophysics. Well-established astrophysical populations, whose brightest members have been robustly detected, represent guaranteed components to the EGB. Among these, the extragalactic components are blazars, radio galaxies, and star-forming galaxies (see Fornasa \& Sánchez-Conde 2015, for a review).

Blazars are among the brightest $\gamma$-ray emitters in the sky. They account for $\sim 50_{-11}^{+12} \%$ of the EGB in the energy interval $E_{\gamma} \lesssim 10 \mathrm{GeV}$, and for $\sim 85_{-21}^{+15} \%$ at $E_{\gamma} \gtrsim 10 \mathrm{GeV}$ (Ajello et al. 2015). They are interpreted as active galactic nuclei (AGN) with the relativistic jet directed towards the observer. The $\gamma$-ray emission in blazars is produced by inverse Compton (IC) scattering of the electrons accelerated in the jet and either the synchrotron photons emitted by the same leptonic population (synchrotron-self Compton), or from accretion disk photons (external Compton).

According to the AGN unification model (Antonucci \& Miller 1985) the viewing angle discriminates among blazars and radio galaxies. With no doppler boost, radio galaxies are expected to be less bright but more abundant than blazars (blazars represent $\sim 10 \%$ of the AGN population), making them potentially important contributors to the EGB. However their contribution to EGB is not well constrained ranging from $\sim 7 \%$ to $\sim 30 \%$ of the EGB intensity measured by 
Fermi-LAT at $E_{\gamma} \lesssim 10 \mathrm{GeV}$ (Inoue 2011; Di Mauro et al. 2014; Wang \& Loeb 2016a).

Recently, the AGN population that do not exhibit relativistic jets have also been considered as possible astrophysical contributors to the EGB (Tamborra et al. 2014; Wang \& Loeb 2016a). In fact, several pieces of observational evidence indicate that AGN produce wide-angle winds with velocities of $v \sim 0.1-$ 0.3 c (e.g. Chartas et al. 2002; Pounds et al. 2003; Reeves et al. 2003; Tombesi et al. 2010, 2015). The shocks produced by the interaction of AGN winds with the ambient medium are expected to accelerate particles to relativistic energies, and the interactions of shock-accelerated particles with surrounding ISM and interstellar radiation field can produce non-thermal emission in the $\gamma$-ray band (Nims et al. 2015; Wang \& Loeb 2016a; Lamastra et al. 2016).

The same emission mechanisms are expected to produce $\gamma$ rays in star-forming galaxies. In this case, the shocks are produced by supernovae (SN) explosions following star formation. Two modes of star formation have been observationally identified: a quiescent mode where the star formation is extended over the whole galactic disk and occurs on time scales of (1-2) Gyr; and a starburst mode where the star formation is concentrated in the dense, nuclear region of galaxies, and it is sustained at an enhanced rate in comparison to quiescently star-forming galaxies. There are several studies that derive the contribution to the EGB from star-forming galaxies (e.g. Fields et al. 2010; Stecker \& Venters 2011; Makiya et al. 2011; Ackermann et al. 2012; Chakraborty \& Fields 2013; Lacki et al. 2014; Tamborra et al. 2014). The studies that analyse quiescent and starburst galaxies separately find that the starburst contribution is always comparatively minor and the total (quiescent plus starburst) $\gamma$-ray emission is between $10 \%$ and $50 \%$ of the EGB intensity.

However, large uncertainties remain for the contribution to the EGB of the above source classes. In this paper we present improved modelling of the integrated $\gamma$-ray emission from AGN winds and star-forming galaxies by incorporating a physical model for the $\gamma$-ray emission produced by particles accelerated in AGN-driven and SN-driven shocks (Lamastra et al. 2016) into a state-of-the-art semi-analytic model (SAM) of galaxy formation (Menci et al. 2014). Our SAM includes a physical description of starburst and AGN activities triggered by galaxy interactions during their merging histories, and is ideally suited for this goal as it has been tested against several observational properties of the AGN and galaxy population both in the local and high-redshift universe, and in different electromagnetic bands (e.g. Menci et al. 2005, 2006; Lamastra et al. 2010, 2013a,b; Menci et al. 2014; Gatti et al. 2015). Moreover, galaxy and AGN number densities, and galaxy properties that determine the $\gamma$-ray emission, like gas mass, star formation rate (SFR), and AGN bolometric luminosity, can be calculated self-consistently by our SAM. This represents an advantage of the semi-analytic approach with respect to previous studies based on parametric expressions for the evolution of the AGN and galaxy populations (derived from observations in a particular electromagnetic band), and on simple scaling laws to relate the $\gamma$-ray luminosity with the properties of the host galaxies.

The paper is organised as follows. Section 2 describes the physical processes producing $\gamma$-ray emission in AGN-driven and $\mathrm{SN}$-driven shocks. A description of the SAM is given in Sect. 3. Section 4 describes how we model the $\gamma$-ray emission from AGN winds and star-forming galaxies. In Sect. 5 we derive the contribution to the EGB from AGN winds and star-forming galaxies, and the Discussion and Conclusions follow in Sects. 6 and 7.

\section{Gamma-ray emission from astrophysical shocks}

The standard paradigm for the origin of the $\gamma$-ray emission in star-forming galaxies is non-thermal emission from relativistic particles accelerated in the shocks produced by SN explosions. Similarly to the shocks surrounding SN remnants, the shocks produced by the interaction of AGN winds with the surrounding ISM are expected to accelerate particles to relativistic energies (Nims et al. 2015; Wang \& Loeb 2016a; Lamastra et al. 2016). In fact, outflows of ionised, neutral, and molecular gas, extended from a few milli-pc to $\mathrm{kpc}$ scales from the central supermassive black hole (SMBH), are now commonly observed in local and high-redshift AGN (see Fiore et al. 2017, and references therein). The most powerful of these AGN winds are made by fast $(v \sim 0.1-0.3 c)$ highly ionised gas particles that are likely accretion disc particles accelerated by the AGN radiation field. The shock pattern resulting from the impact of an AGN wind on the ISM gas is similar to that of the stellar wind hitting the ISM around it (e.g. Weaver et al. 1977; King \& Pounds 2015; King 2003; King \& Pounds 2003; King et al. 2011; Lapi et al. 2005; Faucher-Giguère \& Quataert 2012; Zubovas \& King 2012; Zubovas \& Nayakshin 2014). The wind-ISM interaction is expected to drive an outer forward shock into the ISM accelerating the swept-up material, and an inner reverse shock into the wind decelerating itself, separated by a contact discontinuity. The cooling properties of the shocked wind gas determines whether the outflow is energy- or momentumdriven. In the limit of efficient cooling of the shocked wind gas, most of the pre-shock kinetic energy is radiated away, and only its momentum flux is transferred to the ISM (momentumdriven). In contrast, if the shocked wind gas does not cool, all the energy initially provided by the shock is retained within the systems, and the shocked wind gas expands adiabatically, pushing the ISM gas away (energy-driven).

Inelastic collisions between $\mathrm{CR}$ protons accelerated by AGN-driven and $\mathrm{SN}$-driven shocks with ambient protons may produce a significant $\gamma$-ray emission. In fact, inelastic protonproton collisions produce neutral and charged pions. Neutral pions decay into two $\gamma$-rays: $\pi^{0} \rightarrow \gamma+\gamma$; while charged pions decay into secondary electrons and positrons and neutrinos: $\pi^{+} \rightarrow \mu^{+}+v_{\mu}$ and $\mu^{+} \rightarrow \mathrm{e}^{+}+v_{\mathrm{e}}+\bar{v}_{\mu} ; \pi^{-} \rightarrow \mu^{-}+\bar{v}_{\mu}$ and $\mu^{-} \rightarrow \mathrm{e}^{-}+\bar{v}_{\mathrm{e}}+v_{\mu}$. CR electrons can also produce $\gamma$-ray emission either through interaction with ISM gas (bremsstrahlung) or interstellar radiation field (IC scattering).

In our previous paper (Lamastra et al. 2016) we developed a physical model for the $\gamma$-ray emission from relativistic protons and electrons accelerated by astrophysical shocks. This model was used to predict the $\gamma$-ray spectrum produced by CR particles accelerated by the shocks observed in the molecular disk of the Seyfert galaxy NGC 1068. In this paper we derive the $\gamma$-ray emission from AGN winds and star-forming galaxies in a cosmological context by including the physical model for the $\gamma$-ray emission into a semi-analytic model of hierarchical galaxy formation. Our aim is to compare the model predictions with the measurement of the EGB intensity performed by the Fermi-LAT in the range between $100 \mathrm{MeV}$ and $820 \mathrm{GeV}$ (Ackermann et al. 2015). In this energy range leptonic $\gamma$-ray emission is expected to be lesser than the hadronic one; thus we limit the calculation of the $\gamma$-ray spectrum to the hadronic component.

Here we briefly recall the basic points of our model. We assume that protons are accelerated by diffusive shock acceleration (DSA) to relativistic energies in the forward outflow shock. The resulting proton number density per unit volume can be expressed as a power-law with spectral index 
$p \simeq 2$ and an exponential high-energy cut-off (Bell 1978a,b; Blandford \& Ostriker 1978; Drury 1983):

$N\left(E_{\mathrm{p}}\right)=A_{\mathrm{p}} E_{\mathrm{p}}^{-p} \exp \left[-\left(\frac{E_{\mathrm{p}}}{E_{\max }}\right)\right]$.

The normalisation constant $A_{\mathrm{p}}$ is determined by the total energy supplied to relativistic protons at the shock, and $E_{\max }$ is the maximum energy of accelerated protons. The latter can be obtained by equating the proton acceleration time $\tau_{\text {acc }}=E_{\mathrm{p}} c / e B v_{\mathrm{s}}^{2}$, where $e$ is the electron charge, $v_{\mathrm{s}}$ is the shock velocity, and $B$ is the magnetic field strength, to either the time scale of proton-proton collisions $\tau_{\mathrm{pp}} \approx 5 \times 10^{7} \mathrm{yr} /\left(n_{\mathrm{H}} / \mathrm{cm}^{-3}\right)$, where $n_{\mathrm{H}}$ in the ISM number density, or the outflow time scale $\tau_{\mathrm{s}}=R_{\mathrm{s}} / v_{\mathrm{s}}$. Thus:

$E_{\max }=0.5 v_{s, 8}^{2} \tau_{\text {age }, 3} B_{\mu \mathrm{G}} \mathrm{TeV}$,

where $v_{\mathrm{s}, 8}$ is the shock velocity in units of $10^{8} \mathrm{~cm} / \mathrm{s}, \tau_{\mathrm{age}, 3}$ is the age of the accelerator in units of $10^{3} \mathrm{yr}$, and $B_{\mu \mathrm{G}}$ is the magnetic field strength in units of $\mu \mathrm{G}$ (Reynolds 2008).

We constrain the normalisation constant $A_{\mathrm{p}}$ in Eq. (1) as:

$\int_{E_{\min }}^{E_{\max }} N(E) E \mathrm{~d} E=\eta_{\mathrm{p}} E_{\mathrm{kin}}$,

where $E_{\min }=m_{\mathrm{p}} c^{2}$ is the minimum energy of an accelerated proton which is set to be proton rest mass, $E_{\text {kin }}$ is the kinetic energy of the shocked particles, and $\eta_{\mathrm{p}}$ is the fraction of the kinetic energy transferred to protons. For the latter, we adopt $\eta_{\mathrm{p}} \simeq 0.1$, that is, the value assumed in standard $\mathrm{SN}$-driven shocks (Keshet et al. 2003; Thompson et al. 2006; Tatischeff 2008; Lacki et al. 2010).

We compute the $\gamma$-ray spectrum produced by neutral pion decay using the $\delta$-functional approximation (Stecker 1970; Aharonian \& Atoyan 2000):

$L_{\gamma}(E)=2 V E^{2} \int_{E_{\min }}^{\infty} \frac{q_{\pi}\left(E_{\pi}\right)}{\left(E_{\pi}^{2}-m_{\pi}^{2} c^{4}\right)^{0.5}} \mathrm{~d} E_{\pi}$,

where $V$ is the volume of the outflow, $E_{\min }=E+m_{\pi}^{2} c^{4} / 4 E$, and $E_{\pi}$ and $m_{\pi}$ are the energy and mass of the neutral pion. The emissivity of $\pi^{0}$ is given by:

$q_{\pi}\left(E_{\pi}\right)=\frac{c n_{\mathrm{H}}}{k_{\mathrm{pp}}} \sigma_{\mathrm{pp}}(x) N(x)$,

where $x=m_{\mathrm{p}} c^{2}+E_{\pi} / k_{\mathrm{pp}}, k_{\mathrm{pp}}=0.17$ is the fraction of the accelerated proton energy that goes to neutral pions in each interactions, $\sigma_{\mathrm{pp}}$ is the inelastic cross-section of a proton-proton collision, and $N(x)$ is the accelerated proton energy distribution.

\section{The semi-analytic model}

In order to connect the above modelling of source emission to the statistical description of galaxy and AGN populations in a cosmological framework, we use the SAM described in detail in Menci et al. (2014; see also Gatti et al. 2015).

\subsection{Hierarchical galaxy formation and SMBH growth}

The SAM connects the cosmological evolution of dark matter halos with the processes involving their baryonic content. An accurate Monte Carlo procedure is used to generate the merging trees of dark matter halos following the Press \& Schecter formalism (Bond et al. 1991; Lacey \& Cole 1993), and to describe the gradual inclusion of sub-halos and their dynamical friction processes and binary interactions (major and minor merging and fly-by events).

We assume a cold dark matter power spectrum of perturbations and we adopted a Hubble constant $h=0.7$ in units of $100 \mathrm{~km} \mathrm{~s}^{-1} \mathrm{Mpc}^{-1}$, a dark energy density parameter $\Omega_{\Lambda}=0.7$, a matter density parameter $\Omega_{\mathrm{M}}=0.3$, and a baryon density parameter $\Omega_{\mathrm{b}}=0.035$.

The baryonic processes taking place in each dark matter halo are computed following the standard recipes commonly adopted in SAMs. Starting from an initial amount, $M_{\text {gas }}=M_{\text {halo }} \Omega_{\mathrm{b}} / \Omega_{\mathrm{M}}$, of gas at the virial temperature in each dark matter halos, we compute the mass of cold baryons which are able to radiatively cool. The cooled gas settles into a rotationally supported disk with mass $M_{\mathrm{c}}$, disk circular velocity $v_{\mathrm{d}}$, and disk radius $r_{\mathrm{d}}$ (typically ranging from 1 to $5 \mathrm{kpc}$ ) computed as in Mo et al. (1998).

The cooled gas mass $M_{\mathrm{c}}$ is converted into stars through two different channels: i) quiescent star formation, gradually converting the gas into stars with a rate $S F R=M_{\mathrm{c}} / \tau_{*}$ given by the Schmidt-Kennicutt law with $\tau_{*}=1 \mathrm{Gyr}$; or ii) starbursts following galaxy interactions (merging and fly-bys) occurring on time scales of $\sim 10^{7}-10^{8} \mathrm{yr}$ given by the duration of the interaction.

We assume that all stars with masses in the SN regime explode together, giving rise to a single bubble, and that a fraction of the total energy released by SN explosions is fed back onto the galactic gas. Thus, the effect of $\mathrm{SN}$ feedback is to return part of the cooled gas to the hot phase. The mass $\Delta m_{\mathrm{h}}$ returned from the cold gas content of the disk to the hot gas phase is estimated, at each time-step from canonical energy balance arguments (Kauffmann 1996; Kauffmann \& Charlot 1998) as:

$\Delta m_{\mathrm{h}}=\frac{E_{\mathrm{SN}} \epsilon_{\mathrm{SN}} \phi \Delta m_{*}}{v_{\mathrm{c}}^{2}}$,

where $E_{\mathrm{SN}}=10^{51} \mathrm{erg}$ is the energy of ejecta of each $\mathrm{SN}$, $\epsilon_{\mathrm{SN}}=0.01-0.5$ is the efficiency for the coupling of the emitted energy with the cold ISM, $\phi=0.003-0.005 M_{\odot}^{-1}$ is the number of SN per unit solar mass, depending on the assumed initial mass function (IMF), and $v_{\mathrm{c}}$ is the circular velocity of the galactic halo. The model free parameter $\epsilon_{\mathrm{SN}}=0.1$ is chosen as to match the local $B$-band luminosity function and the TullyFisher relation adopting a Salpeter IMF. Although our simple modelling of SN feedback does not include a detailed treatment of the gas kinematics, including the dynamics of superbubbles (Ferrara et al. 2000), it provides a good match to the observed correlations between the outflow velocity with the galactic circular velocity, and the SFR (Calura \& Menci 2009).

The luminosity produced by the stellar population of the galaxies is computed by convolving the star-formation histories of the galaxy progenitors with a synthetic spectral energy distribution (SED, Bruzual \& Charlot 2003). The dust extinction affecting the above luminosities is computed assuming the dust optical depth to be proportional to the metallicity $Z_{\text {cold }}$ of the cold phase (computed assuming a constant effective yield) and to the disk surface density, so that for the $V$ band $\tau_{V} \propto M_{\mathrm{c}} Z_{\text {cold }} / \pi r_{\mathrm{d}}^{2}$. The proportionality constant is taken as to match the bright end of the local luminosity function. To compute the extinction at other wavelengths we applied a proper extinction curve (see Menci et al. 2002, 2005).

The SAM includes the growth of SMBHs from primordial seeds. The latter are assumed to be the end-product of PopIII stars with a mass $M_{\text {seed }}=100 M_{\odot}$ (Madau \& Rees 2001), and to be initially present in all galaxy progenitors. SMBHs grow by merging with other black holes following the coalescence of the host galaxies and by accretion of cold galactic gas. The latter gives rise to the AGN activity. The gas accretion is triggered 


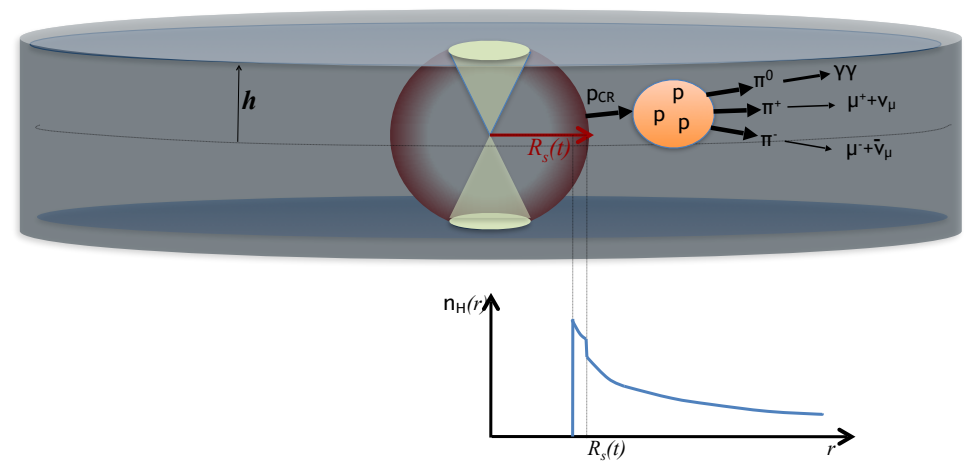

Fig. 1. Schematic representation of the interaction of CR protons accelerated in the AGN blast wave with ISM protons. The shock radius $R_{\mathrm{S}}(t)$ expands outwards compressing the swept gas into a thin shell, and leaving a cavity inside. The $\gamma$-ray emission from neutral pion decays occurs in the regions outside the yellow cones where the lines of sight intercept the galactic gas that has not been swept out by the blast wave.

by galaxy interactions. In particular, we assume the analytical description of the gas inflows induced by galaxy interactions derived by Cavaliere \& Vittorini (2000; see also Menci et al. 2006, 2008; Lamastra et al. 2013b), and that in each galaxy interaction one quarter of the destabilised gas feeds the SMBH, while the remaining fraction feeds the circumnuclear starburst (Sanders \& Mirabel 1996). These gas fractions are calibrated as to yield final SMBH masses matching the observed local correlations with the properties of the host galaxies. We converted the mass accretion rate $\dot{M}_{\mathrm{BH}}$ into AGN bolometric luminosity as:

$L_{\mathrm{AGN}}=\eta \dot{M}_{\mathrm{BH}} c^{2}$,

where $\eta \simeq 0.1$ is the efficiency for the conversion of gravitational energy into radiation (Yu \& Tremaine 2002; Marconi et al. 2004). Our SAM also includes a model of AGN feedback described in detail in Sect. 3.2.

The SAM has been tested against the statistical properties of the galaxy and AGN populations at low and high redshift and in different electromagnetic bands. In particular, the model provides galaxy luminosity functions (LF) in the $K$-band (a proxy for the stellar mass content) and in the $U V$ band (a proxy for the instantaneous SFR) that are in good agreement with the observed evolution of the galaxy LF in the $K$-band up to $z \sim 3$ and with the galaxy LF in the UV band up to $z \sim 6$ (see Menci et al. 2014). The model is also able to reproduce the well known bimodal distribution of galaxies in the colour-magnitude diagram (see Menci et al. 2014).

The model predicts AGN LFs in the UV band that are in good agreement with the observational estimates at intermediate and high luminosities up to $z \sim 6$. At all redshifts, the model tends to slightly overestimate the data at faint luminosities (see Menci et al. 2014). The observational scaling relations between the galaxy and AGN physical properties (such as stellar mass, SFR, SMBH mass, and $\left.\dot{M}_{\mathrm{BH}}\right)$ are also well described by the model (Menci et al. 2005, 2006; Lamastra et al. 2010, 2013a,b; Menci et al. 2014; Gatti et al. 2015).

\subsection{The blast wave model for AGN feedback}

Our SAM includes a physical model for AGN feedback which is related to the impulsive luminous AGN phase. As discussed in Sect. 2, mildly relativistic winds $(v \sim 0.1-0.3 c)$ are injected by AGN into the surrounding ISM (Chartas et al. 2002; Pounds et al. 2003; Reeves et al. 2003; Tombesi et al. 2010, 2015). As these winds propagate into the ISM, they compress the gas into a blast wave terminated by a leading shock front, which moves outward with a lower but still supersonic velocity and sweeps out the surrounding medium. The expansion of the blast wave into the ISM is described by hydrodynamical equations. Taking into account the effect of dark-matter gravity, upstream pressure, and initial density gradient, and assuming the Rankine-Hugoniot boundary condition at the shock, Lapi et al. (2005) derived an analytic expression for the radius $R_{\mathrm{S}}$ of the blast wave in the case of shock expansion in a gas with a powerlaw density profile $\rho \propto r^{-\omega}$, where the exponent $\omega$ is in the range $2 \leq \omega<2.5$ (see also Chevalier 1976, 1982; Weaver et al. 1977; Ostriker \& McKee 1988; Franco et al. 1991).

In Menci et al. (2008) the expression for the shock radius is given in terms of the galactic disk radius, disk velocity, and Mach number $\mathcal{M}=v_{\mathrm{s}} / c_{\mathrm{S}}\left(R_{\mathrm{s}}(t)\right)$ :

$R_{\mathrm{s}}(t)=v_{\mathrm{d}} t_{\mathrm{d}}\left[\frac{5 \pi \omega^{2}}{24 \pi(\omega-1)}\right]^{1 / \omega} \cdot \mathcal{M}^{2 / \omega}\left[\frac{t}{t_{\mathrm{d}}}\right]^{2 / \omega}$.

The Mach number $\mathcal{M}$ is related to ratio between the energy $\Delta E$ injected by the AGN into the surrounding medium and the total thermal energy $E \propto M_{\mathrm{c}}$ of the ISM:

$\mathcal{M}^{2}=1+\Delta E / E$.

Thus the production of weak $(\mathcal{M} \simeq 1)$ or strong shocks $(\mathcal{M} \gg 1)$ depends on the value of $\Delta E$ which is computed as:

$\Delta E=\epsilon_{\mathrm{AGN}} L_{\mathrm{AGN}} \tau_{\mathrm{AGN}}$,

here $\epsilon_{\mathrm{AGN}}$ is the fraction of the AGN bolometric luminosity transferred to the gas in the form of kinetic energy, and $\tau_{\mathrm{AGN}}$ is the duration of the AGN phase.

The blast wave model for AGN feedback was used in our previous papers to explain the distribution of hydrogen column densities in AGN as a function of luminosity and redshift, and to predict hydrogen phoionization rate as a function of redshift (Menci et al. 2008; Giallongo et al. 2012).

\section{Model set up}

In this section we describe the model parameters that we will use in the computation of the $\gamma$-ray emission from AGN winds and star-forming galaxies.

In particular, we define the parameters that describe the $\gamma$ ray spectra of individual AGN winds and star-forming galaxies, and the environment into which the shocks expand. We limit the shock expansion into galactic disks, for which we assume a constant scale height $h_{\mathrm{d}}=100$ pc (Narayan \& Jog 2002; van der Kruit \& Freeman 2011), and an isothermal gas density profile $n_{\mathrm{H}}=n_{\mathrm{H}, 0} / r^{-2}$ (see Fig. 1 ). The constant $n_{\mathrm{H}, 0}$ in the density profile can be constrained by the total gas content in the $\operatorname{disk} M_{\mathrm{c}}$. 


\subsection{Modelling $\gamma$-ray emission from AGN winds}

To derive the $\gamma$-ray spectrum of an individual AGN wind, we need to determine the energy distribution of the particles accelerated in the shocks. The latter is determined by the $\mathrm{CR}$ particle spectral index $p$, the particle maximum energy $E_{\max }$, and by the total energy supplied to relativistic particles at the shock. As discussed in Sect. 2, we assume that DSA is the mechanism which produces $\mathrm{CR}$ protons in AGN-driven shocks (but see Vazza et al. 2015, 2016; van Weeren et al. 2016, for results showing that DSA has difficulty in explaining the observed emissions of particles accelerated in some astrophysical shocks). DSA could result in the production of a power-law accelerated proton population with a power-law index $p \simeq 2$ (Bell 1978a,b; Blandford \& Ostriker 1978; Drury 1983). As the particles diffuse from the acceleration region, energy-dependent diffusion losses can soften the source spectrum leading to larger values of the spectral index $p$. The accelerated particle energy distribution extends to energies as high as is permitted by various loss processes. Protons are accelerated up to a maximum energy that depends on the shock velocity, age of the accelerator, and on the magnetic field strength in the shock region (Eq. (2)). The first two parameters are determined by the hydrodynamics of the shocks and by the density of the galactic disk. For an isothermal gas disk, the blast wave shock radius is given by $R_{\mathrm{s}}=0.9 \mathcal{M} v_{\mathrm{d}} t$ (Eq. (8)), which implies a constant outflow velocity $v_{\mathrm{s}}=\mathrm{d} R_{\mathrm{s}} / \mathrm{d} t=0.9 \mathcal{M} v_{\mathrm{d}}$. For the magnetic field $B$ we assume values bracketed by a minimum value that is given by the volume average ISM magnetic field strength $B_{\text {ISM }}=6 \times\left(\Sigma_{\text {gas }} / 0.0025 \mathrm{~g} \mathrm{~cm}^{-2}\right)^{a} \mu \mathrm{G}$ where $a \simeq 0.4-1$ and $\Sigma_{\text {gas }}=2 n_{\mathrm{H}} m_{\mathrm{H}} h_{\mathrm{d}}$ is the disk gas surface density (Robishaw et al. 2008; Lacki et al. 2010; McBride et al. 2014); and a maximum value that is derived by assuming that a fraction $\xi_{\mathrm{B}} \simeq 0.1$, based on observations of SN remnants (Chevalier 1998), of the post shock thermal energy is carried by the magnetic field $B_{\text {shock }}=\left(8 \pi \xi_{\mathrm{B}} n_{\mathrm{s}} k T_{\mathrm{s}}\right)^{0.5}$ where $n_{\mathrm{s}}$ and $T_{\mathrm{s}}$ are the post-shock density and temperature of the gas, respectively. To derive the maximum value of the magnetic field $B_{\text {shock }}$ we assume the temperature and density jumps given by the approximations valid for very strong shocks as given in Lapi et al. (2005): $n_{\mathrm{s}} \simeq 4 n_{\mathrm{H}}$ and $T_{\mathrm{s}} \simeq \mu m_{\mathrm{p}} v_{\mathrm{s}}^{2} / 3 k$, irrespective of $\mathcal{M}$.

A constant outflow velocity corresponds to an energy-driven outflow in which the kinetic luminosity does not vary in time. In this case, the kinetic energy of accelerated protons is simply given by the product of the outflow kinetic luminosity $L_{\text {kin }}$ and the residence time of the particles in the acceleration region $\tau_{\text {res }}$ :

$E_{\text {kin }}=L_{\text {kin }} \tau_{\text {res }}$

In the case of AGN-driven winds, we assume that the outflow kinetic luminosity is a fraction $\epsilon_{\mathrm{AGN}}$ of the AGN bolometric luminosity:

$L_{\mathrm{kin}}^{\mathrm{AGN}}=\epsilon_{\mathrm{AGN}} L_{\mathrm{AGN}}$.

The ratio between the outflow kinetic power and AGN bolometric luminosity has recently been determined in a sample of 94 AGN by Fiore et al. (2017) to be in the range $\epsilon_{\mathrm{AGN}}=0.001-0.1$. The parameter $\epsilon_{\mathrm{AGN}}=0.01$ is chosen within the observational range as to provide a good fit of the bright end of the AGN luminosity function (see Menci et al. 2014).

The collisions between CR protons and ambient protons in galactic disks produce hadronic $\gamma$-ray emission. The blast wave model for AGN feedback allows us to self-consistently compute

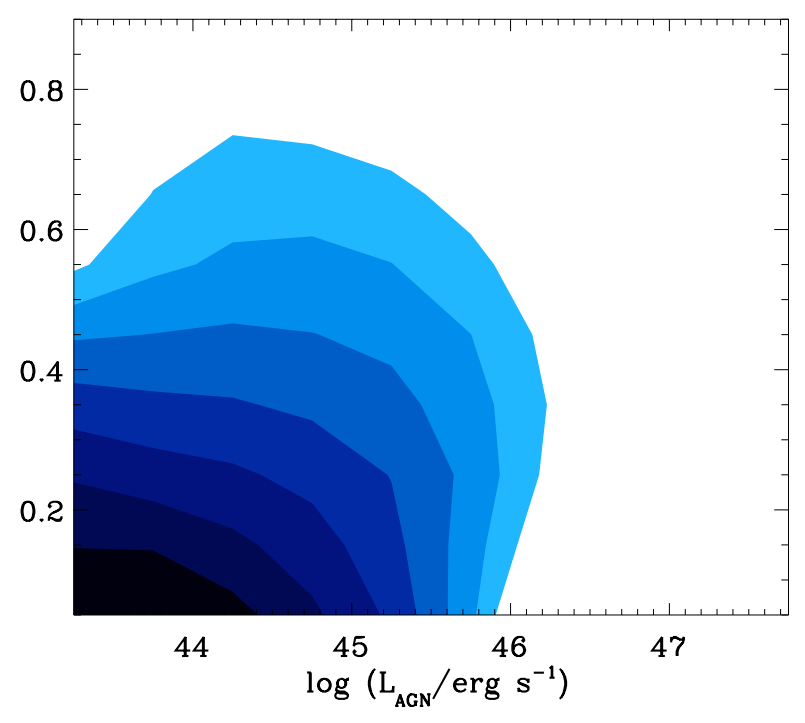

Fig. 2. Distribution of simulated $\mathrm{AGN}$ as a function of $F_{\mathrm{cal}}^{\mathrm{AGN}}$ and $\mathrm{AGN}$ bolometric luminosity for our fiducial model. The contours correspond to equally spaced values of the density (per $\mathrm{Mpc}^{3}$ ) of objects in a given $F_{\text {cal }}^{\mathrm{AGN}}-L_{\mathrm{AGN}}$ bin in logarithmic scale: from $10^{-5}$ for the lightest filled region to $10^{-2}$ for the darkest.

the fraction of accelerated protons that may interact with ambient protons. In fact $\gamma$-ray emission from neutral pion decays occurs along the lines of sight where the galactic gas has not been swept out by the blast wave produced by the AGN (see Fig. 1). This fraction is the complementary part of the escape fraction of ionising photons that we derived in Giallongo et al. (2012). The average of this fraction over the duration $\tau_{\mathrm{AGN}}$ of the AGN activity is given by:

$F_{\mathrm{cal}}^{\mathrm{AGN}}=\frac{\tau_{\mathrm{s}}}{\tau_{\mathrm{AGN}}}\left[1-\ln \left(\frac{\tau_{\mathrm{s}}}{\tau_{\mathrm{AGN}}}\right)\right]$,

where $\tau_{\mathrm{s}}$ is the time at which the shock radius first encompasses the width of the galactic disk: $\tau_{\mathrm{s}}=h_{\mathrm{d}} / 0.9 \mathcal{M} r_{\mathrm{d}}$. For shocks with high Mach number, that is, for large AGN injected energies $\Delta E \gg E, \tau_{\mathrm{s}} \ll \tau_{\mathrm{AGN}}$ yielding small fractions of interacting protons. Figure 2 shows the distribution of simulated AGN as a function of $F_{\text {cal }}^{\mathrm{AGN}}$ and AGN bolometric luminosity for our fiducial model.

We assume that all the energy of the protons that interact with the protons in the galactic disk is converted into pion production. This corresponds to an assumtion that AGN winds act as proton calorimeters. The calorimetric limit has the maximum efficiency to convert AGN blast wave energy into $\gamma$-rays, and this corresponds to the assumption that $\tau_{\text {res }}=\tau_{\text {pp }}$ in Eq. (11). The proton-proton collisional time scale $\tau_{\mathrm{pp}}$ is inversely proportional to the density $n_{\mathrm{H}}$ of target material, thus the resulting hadronic $\gamma$-ray luminosity is independent of $n_{\mathrm{H}}$, and it scales linearly with the outflow kinetic luminosity (see Eq. (4)). Figure 3 shows the predicted $\gamma$-ray spectrum of an AGN with $L_{\mathrm{AGN}}=7 \times 10^{44} \mathrm{erg} / \mathrm{s}$ hosted in a halo of mass $10^{12} M_{\odot}$ at $z=0.1$. In our derivation of the $\gamma$-ray spectrum we neglect the $\gamma$-ray emission from IC and bremsstrahlung processes of the primary and secondary leptonic populations, as it is expected to be lesser than the hadronic one at $E_{\gamma} \gtrsim 100 \mathrm{MeV}$ (Lacki et al. 2014). 


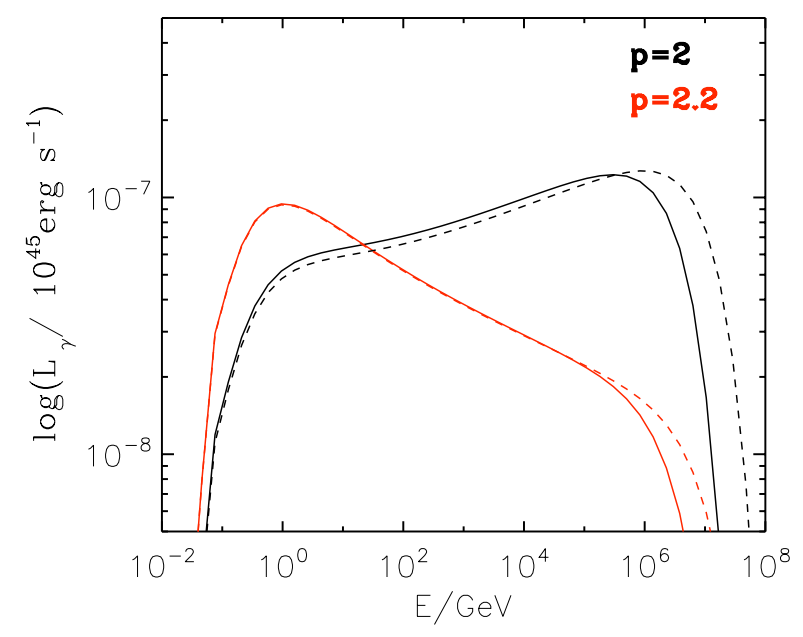

Fig. 3. $\gamma$-ray spectrum of an AGN with $L_{\mathrm{AGN}}=7 \times 10^{44} \mathrm{erg} / \mathrm{s}$ hosted in a halo of mass $10^{12} M_{\odot}$ at $z=0.1$. Energy spectrum parameters are set at $p=2$ (black lines), $p=2.2$ (red lines), $B=B_{\mathrm{ISM}}$ (solid lines), and $B=B_{\text {shock }}$ (dashed lines).

\subsection{Modelling $\gamma$-ray emission from star-forming galaxies}

Besides the Milky Way, ten external star-forming galaxies have been firmly detected in $\gamma$-rays with the Fermi-LAT (Ackermann et al. 2012, 2017). Among these, seven are starburst and active galaxies that are more luminous at $\gamma$-ray energies compared to quiescently star-forming galaxies by a factor larger than 10. The $\gamma$-ray spectra of starbursts look similar and can be described by a single power-law with spectral index $p \sim 2.2$. Because of the high density of star-forming regions in starburst galaxies, it is likely that they act as proton calorimeters (Wang \& Fields 2016). Thus starburst galaxies could have harder spectra than quiescent galaxies as in their acceleration region proton energy losses are dominated by nearly energyindependent proton-proton collisions. On the contrary, in quiescent galaxies proton losses are thought to be dominated by energy-dependent diffusion (as in the Milky Way), and their $\gamma$-ray luminosity is set by equilibrium between proton injection rate, diffusion processes, and energy losses. Both kinds of starforming galaxy are included in our SAM. However, for the above reasons, modelling the detailed escape and energy-loss process of accelerated protons in quiescent galaxies is a very difficult task. Thus, in the following we consider only the calorimetric regime, which provides a good description of starburst galaxies, and we discuss the implications for the $\gamma$-ray emission from the more numerous but less powerful quiescient galaxies.

To derive the energy distribution of particles accelerated in starburst galaxies we assume a power-law index $p=2.2$ characteristic of the starbursts detected in the $\gamma$-ray band (Ackermann et al. 2012). We also assume a constant shock velocity equal to $v_{\mathrm{s}}=300 \mathrm{~km} \mathrm{~s}^{-1}$ (Lacki et al. 2010), and a minimum and maximum value of the magnetic field in the shock region given by $B_{\text {ISM }}$ and $B_{\text {shock }}$, respectively, as in the case of AGN-driven shocks. The kinetic luminosity available in the form of accelerated protons for a starburst galaxy is:

$L_{\mathrm{kin}}^{\mathrm{SB}}=\epsilon_{\mathrm{SN}} \nu_{\mathrm{SN}} E_{\mathrm{SN}}$,

where $v_{\mathrm{SN}}$ is the $\mathrm{SN}$ rate, and $\epsilon_{\mathrm{SN}}=0.1$ is the fraction of the $\mathrm{SN}$ energy transferred to the gas in the form of kinetic energy (see Sect. 3). SN rates can be estimated from SFR and the IMF as $v_{\mathrm{SN}}=\phi \times S F R$ where $\phi \simeq 0.003 M_{\odot}^{-1}$ for a Salpeter IMF.
We note that for starburst galaxies in the calorimetric regime the $\gamma$-ray luminosity scales linearly with the SFR.

Starburst galaxies are selected from the SAM as model galaxies dominated by the SFR triggered by galaxy interactions. The latter at the same time trigger AGN activity. The starburstAGN connection predicted by the SAM (see Lamastra et al. 2013 b) implies that the blast wave produced by the AGN could sweep out the disk gas when the starbursts are in action. This hampers the derivation of the fraction of protons accelerated in $\mathrm{SN}$-driven shocks that interact with protons in the ISM. In fact, the latter depends on the time delay between the trigger of the AGN and starburst activities, and on the starburst spatial distribution quantity that cannot be provided by the SAM. For this reason, in the following, to derive the contribution from starburst galaxies to the EGB, we adopt an empirical calorimetric fraction, which is derived from observations of star-forming galaxies in the $\mathrm{GeV}$ band (Ackermann et al. 2012):

$$
F_{\mathrm{cal}}^{\mathrm{SB}}=0.3\left(\frac{S F R}{M_{\odot} \mathrm{yr}^{-1}}\right)^{0.16}\left(\frac{E_{\mathrm{SN}}}{10^{51} \mathrm{erg}}\right)^{-1}\left(\frac{\eta_{\mathrm{p}}}{0.1}\right)^{-1}
$$

\section{Results}

In this section we derive the cumulative $\gamma$-ray emission from AGN winds and starburst galaxies predicted by the SAM. The contribution from AGN winds (starburst galaxies) to the EGB spectrum can be estimated as:

$$
\begin{aligned}
E^{2} \frac{\mathrm{d} N}{\mathrm{~d} E}= & \int_{0}^{z_{\max }} \int_{L_{\gamma, \min }}^{L_{\gamma, \max }} \phi\left(L_{\gamma}, z\right) \frac{I\left(E_{\gamma}^{\prime}, L_{\gamma}, z\right)}{4 \pi D_{L}^{2}(z)} \\
& \times \exp \left[-\tau_{\gamma \gamma}\left(E_{\gamma}^{\prime}, z\right)\right] \frac{\mathrm{d}^{2} V}{\mathrm{~d} z \mathrm{~d} \Omega} \mathrm{d} L_{\gamma} \mathrm{d} z
\end{aligned}
$$

where $E_{\gamma}^{\prime}=E_{\gamma}(1+z)$ is the intrinsic photon energy, $\phi\left(L_{\gamma}, z\right)$ is the comoving number density of AGN (starburst galaxies) per unit of $\gamma$-ray luminosity as a function of redshift, $D_{L}(z)$ is the luminosity distance, $I\left(E_{\gamma}, L_{\gamma}, z\right)$ is the $\gamma$-ray spectrum of an individual AGN (starburst galaxy) with integral $\gamma$-ray luminosity $L_{\gamma}$ at redshift $z$, the factor $\mathrm{d}^{2} V / \mathrm{d} z \mathrm{~d} \Omega$ represents the comoving volume element per unit redshift and unit solid angle, and $\tau_{\gamma \gamma}$ is the diffuse extragalactic background light (EBL) optical depth for photons with energy $E_{\gamma}$ at a redshift of $z$. In fact, the emitted $\gamma$-rays, while travelling through the inter galactic medium, interact with the photons of EBL and become absorbed through $\mathrm{e}+/ \mathrm{e}-$ pair production. The absorption probability increases with energy and distance of the $\gamma$-ray source. In our calculation we assume $z_{\max }=5$ and adopt the EBL model of Stecker et al. (2016).

Figure 4 compares the cumulative $\gamma$-ray emission from AGN winds and starburst galaxies predicted by the SAM with the EGB spectrum measured by Fermi-LAT (Ackermann et al. 2015). The latter is well described by a power-law with a spectral index of $\sim 2.3$ and an exponential cut-off. Both the statistical and systematic uncertainties of the EGB measurement are shown in Fig. 4. The systematic uncertainty ranges between a factor $\sim 15 \%$ and $\sim 30 \%$ (depending on the energy range considered) and depends on the modelling of the Galactic diffuse emission. The model predictions are shown for our fiducial model corresponding to $h_{\mathrm{d}}=100 \mathrm{pc}, \eta_{\mathrm{p}}=0.1, \epsilon_{\mathrm{AGN}}=0.01$, and $\epsilon_{\mathrm{SN}}=0.1$. The accelerated proton spectral index is assumed to be $p=2.2$ for both AGN winds and starburst galaxies. We find that our results do not depend on the exact value of the magnetic field. In fact, the magnetic field determines the cut-off energy of the EGB spectrum that is mainly affected by EBL attenuation at the 


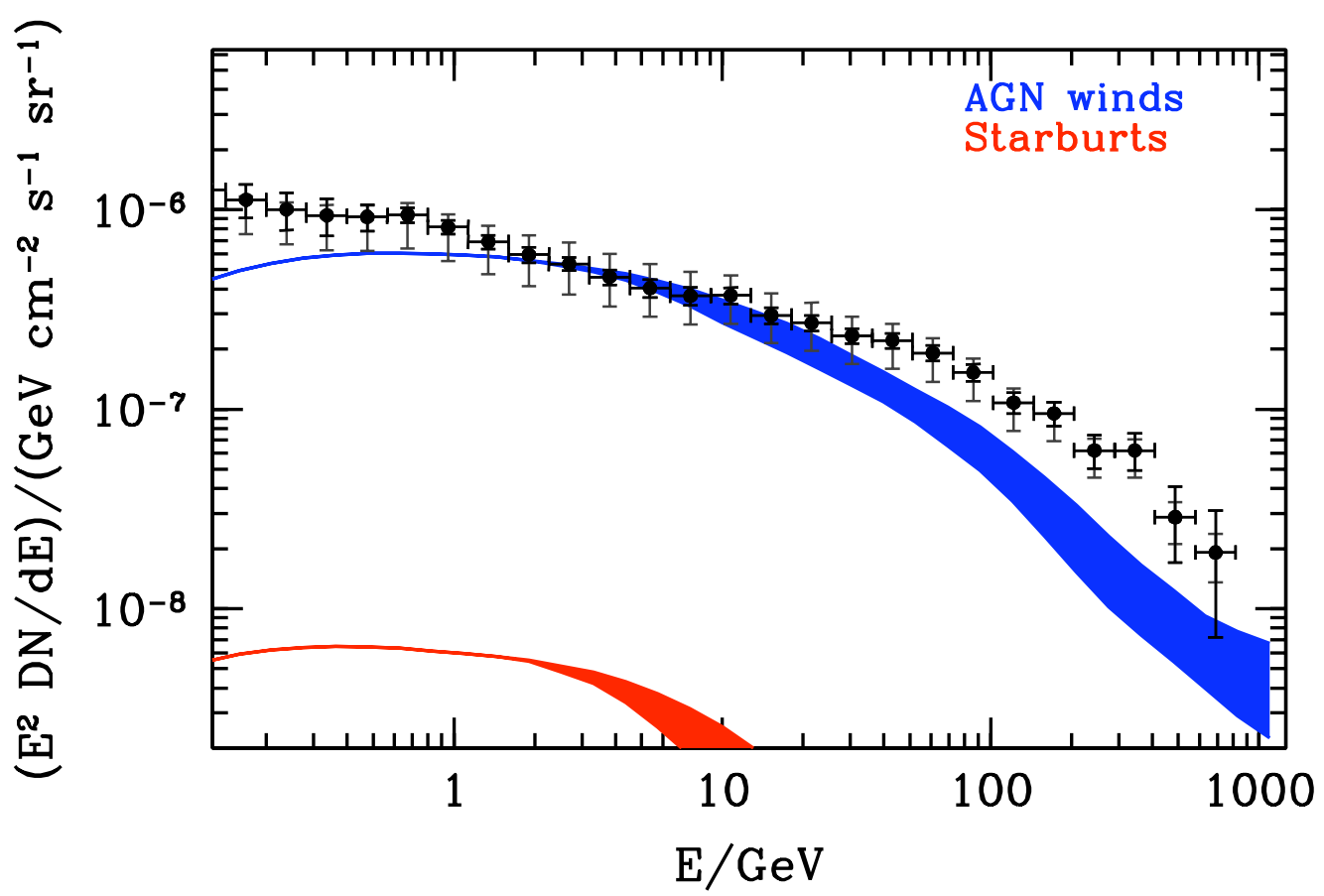

Fig. 4. The contribution to the EGB from AGN winds (blue) and starburst galaxies (red) predicted by our SAM. Fiducial model parameters are set at: $h_{\mathrm{d}}=100 \mathrm{pc}, \eta_{\mathrm{p}}=0.1, \epsilon_{\mathrm{AGN}}=0.01$, and $\epsilon_{\mathrm{SN}}=0.1$. Accelerated proton spectral index equal to $p=2.2$ is assumed for both AGN winds and starburst galaxies. The blue and red shaded bands represent the uncertainty related to the EBL model adopted (Stecker et al. 2016). The data points are the Fermi-LAT measurement of the EGB, with black and grey vertical error bars indicating EGB statistical and systematic uncertainties, respectively (Ackermann et al. 2015).

bright end. Figure 4 shows that, assuming a comparable efficiency for accelerating protons in AGN-driven and SN-driven shocks $\left(\eta_{\mathrm{p}}=0.1\right)$, hierarchical scenarios predict a contribution to the EGB from AGN winds larger by about a factor of 100 than that provided by starburst galaxies. This implies that the kinetic energy available to accelerate protons in AGN winds significantly exceeds that provided by star formation driven by galaxy interactions. However, in hierarchical clustering scenarios starburst galaxies account for a small fraction of the cosmic star formation rate density (SFRD), that is, the mass converted into stars per unit time and comoving volume. The starburst contribution to the SFRD was estimated to be $\sim 5 \%$ at $z \simeq 0.1$ and $\sim 20 \%$ at $z \simeq 5$ (Lamastra et al. 2013a). Thus, the cosmic SFRD is dominated by quiescent galaxies at all redshifts. If we assume that, at all redshifts, the $\gamma$-ray emission scales as the cosmic SFRD, we find that the contribution to the EGB from quiescent galaxies should be a factor of $\sim 20$ greater than that provided by starburst galaxies. This implies that, in hierarchical scenarios, the $\gamma$ ray emission from AGN winds dominates over that powered by star-forming galaxies (quiescent plus starburst). We note that the above order-of-magnitude estimate for the quiescent contribution to the EGB should be thought of as an upper bound. In fact, as discussed in Sect. 4.2, diffusive and advective losses cannot be neglected in quiescent galaxies, thus the hypothesis of proton calorimeter adopted for starburst galaxies, which has the maximum efficiency to convert SN blast wave into $\gamma$-rays, should not be valid in quiescent galaxies.

We find that AGN winds account for $\sim 40 \%$ of the observed EGB in the energy interval $E_{\gamma}=0.1-1 \mathrm{GeV}$, for $\sim 90 \%$ at $E_{\gamma}=1-10 \mathrm{GeV}$, and for $\sim 70 \%$ at $E_{\gamma} \gtrsim 10 \mathrm{GeV}$. Other classes of sources are known to contribute to the EGB. Among these, the major contribution is from blazars. The predicted cumulative emission of blazars is shown in Fig. 5 as a green dashed band (Ajello et al. 2015). The latter encompasses systematic uncertainties on blazar luminosity function models and energy spectrum models. We also show the integrated emission from AGN winds predicted by our SAM assuming different values of the accelerated proton spectral index. As shown in the bottom panel of Fig. 5, at energies $E_{\gamma} \lesssim 10 \mathrm{GeV}$, and for spectral index $p>2$, the $\gamma$-ray emission from AGN winds dominates over that from blazars. The AGN wind contribution to the EGB is peaked at energies $E_{\gamma} \simeq 1-10 \mathrm{GeV}$, depending on the value of $p$, while the blazar contribution reachs its maximum at larger energies $E_{\gamma} \simeq 60 \mathrm{GeV}$. This analysis shows that AGN winds and blazars can account for the amplitude and spectral shape of the EGB, leaving only little room for other contributions.

\section{Discussion}

\subsection{Robustness and comparison with previous works}

Here we discuss our assumptions and compare our results with previous estimates of the $\gamma$-ray emission from AGN winds and star-forming galaxies.

The results shown in the previous section were obtained assuming: i) energy-driven winds powered by the active nucleus in AGN host galaxies; ii) the calorimetric regime; and iii) shock expansion limited to galactic disks.

As for the first point, the observations of winds are very common in AGN host galaxies. X-ray and UV emission and absorption line studies revealed outflows of highly ionised gas on an $\sim 0.001-1$ pc scale, with velocities $v=0.1-0.3 c$, both at low and high redshift (see e.g. Tombesi et al. 2013, 2015). On larger scales (100-1000 pc), outflows of ionised, neutral atomic, and molecular gas, with velocities $v=10^{2}-10^{3} \mathrm{~km} \mathrm{~s}^{-1}$, have been 


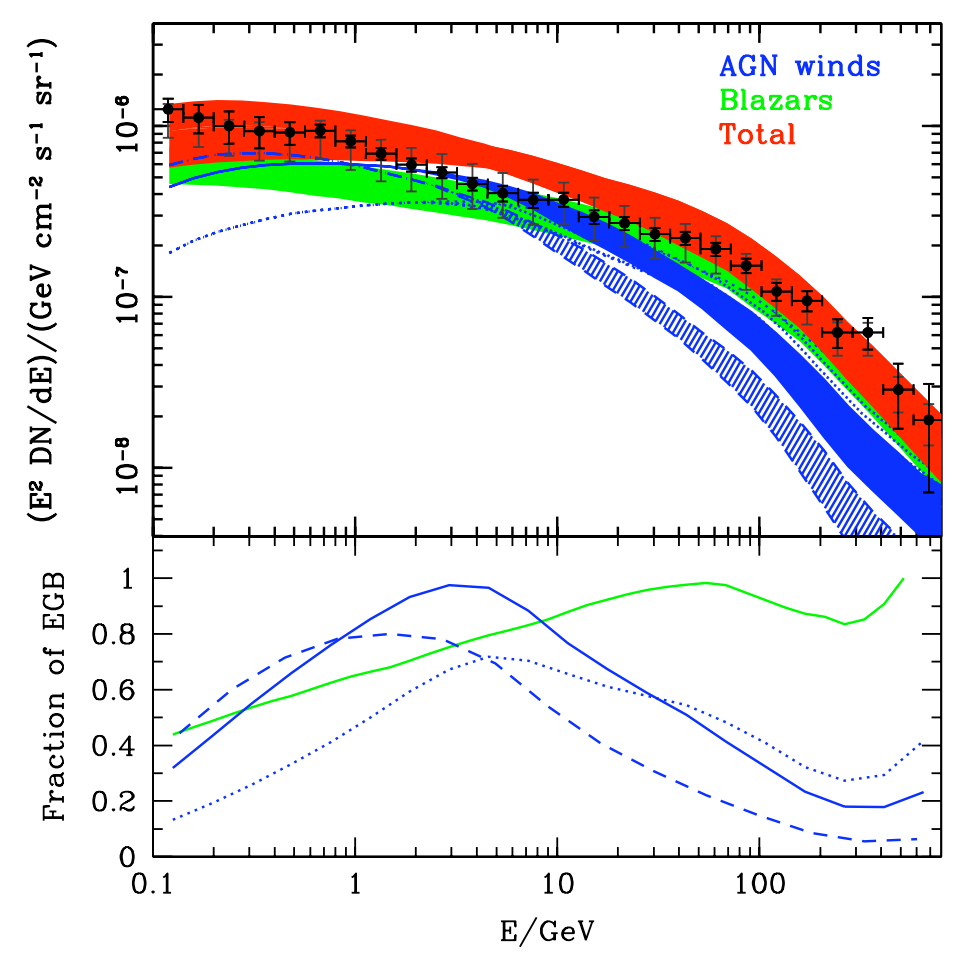

Fig. 5. Upper panel: the contribution to the EGB from AGN winds predicted by our SAM is compared to the contribution provided by the blazar population (Ajello et al. 2015). Model predictions are shown for our fiducial model and for different accelerated proton spectral index: $p=2$ (dotted blue lines), $p=2.2$ (solid blue lines), and $p=2.4$ (dashed blue lines). The red shaded region represent the sum of the AGN wind and blazar contributions to the EGB. The data points are as in Fig. 4. Lower panel: fraction of EGB provided by AGN winds and blazars.

observed through deep optical/near-infrared spectroscopy and interferometric observations in the (sub)millimetre band (e.g. Rupke \& Veilleux 2011; Feruglio et al. 2010, 2015; Cicone et al. 2014; Harrison et al. 2016; Shen 2016; Zakamska et al. 2016). A collection of AGN winds detected at different scales and ionisation states is given in Fiore et al. (2017). These observations indicate that the majority of the large-scale outflows are driven by the nuclear activity, and may be identified with the energydriven phase. For two sources in particular, namely MrK 231 (Feruglio et al. 2015) and IRAS F11119 (Tombesi et al. 2015), both X-ray winds and molecular winds have been detected. The comparison between the momentum rate of the X-ray and molecular winds indicates that these winds are energy-driven. These findings seem to support our assumptions about the frequency and nature of AGN winds, however, these observations are sparse, and mostly limited to AGN selected to have high chances of being in an outflowing phase. In order to gain more insight into these topics, the measurements of the frequency and parameters of AGN winds in unbiased AGN samples over a large range of redshifts are necessary.

As for the calorimetric regime, our model assumes that advective and diffusive escape of accelerated protons in galactic disks are negligible. This condition is satisfied if the protonproton collisional time scale $\tau_{\mathrm{pp}}$ is less than the advective and diffusion time scales. The wind advection time is $\tau_{\text {wind }} \simeq h_{\mathrm{d}} / v_{\mathrm{s}}$. Assuming the velocities measured in galactic scale AGN winds, and for $h_{\mathrm{d}}=100 \mathrm{pc}$, we expect that hadronic losses dominate advection losses when $n_{\mathrm{H}} \gtrsim 50-500 \mathrm{~cm}^{-3}$, which are values observed in circumnuclear disks of active and starburst galaxies (e.g. García-Burillo et al. 2014; Yoast-Hull et al. 2014). The particle diffusion processes in the environments of active and starburst galaxies are poorly constrained. For this reason we neglect diffusive losses, and this could constitute a source of uncertainty in our computation.

As for the environment into which the shocks expand, we limit the expansion to galactic disks, however it is also possible that shocks can propagate in gaseous haloes of galaxies. The lower densities of galactic haloes with respect to galactic disks imply a major role of escape (advective and diffusive) with respect to hadronic interactions, and therefore a low $\gamma$-rayproduction rate. As a check, we can compare our predictions with other recent predictions of the EGB from AGN winds that assume shock expansion in both the disk and halo components of galaxies. Wang \& Loeb (2016a) derived the cumulative $\gamma$-ray emission from AGN winds using a hydrodynamical model for AGN wind interaction with the ambient medium (Wang \& Loeb 2015), and the empirical AGN bolometric luminosity function of Hopkins et al. (2007). In their computation, the $\gamma$-ray emission is assumed to be produced by the same hadronic processes considered in this work and described in Sect. 2.

Figure 6 compares the estimates for the cumulative $\gamma$-ray emission from AGN winds derived by Wang \& Loeb (2016a) with that predicted by our SAM assuming the same fraction of the AGN bolometric luminosity that powers the winds, and the same energy spectrum. Figure 6 shows that the normalisation of the EGB spectrum predicted by the SAM is a factor of $\sim 3$ larger than that derived by Wang \& Loeb (2016a). The SAM also predicts a different shape of the EGB spectrum at energies $E_{\gamma} \gtrsim 1 \mathrm{GeV}$. As for the normalisation, we have checked whether the mismatch seen in Fig. 6 stems from the different AGN luminosity functions adopted. Wang \& Loeb (2016a) used the AGN bolometric luminosity function of Hopkins et al. (2007) which is derived by combining measurements of AGN number density in IR, optical, and X-ray bands, in the redshift 


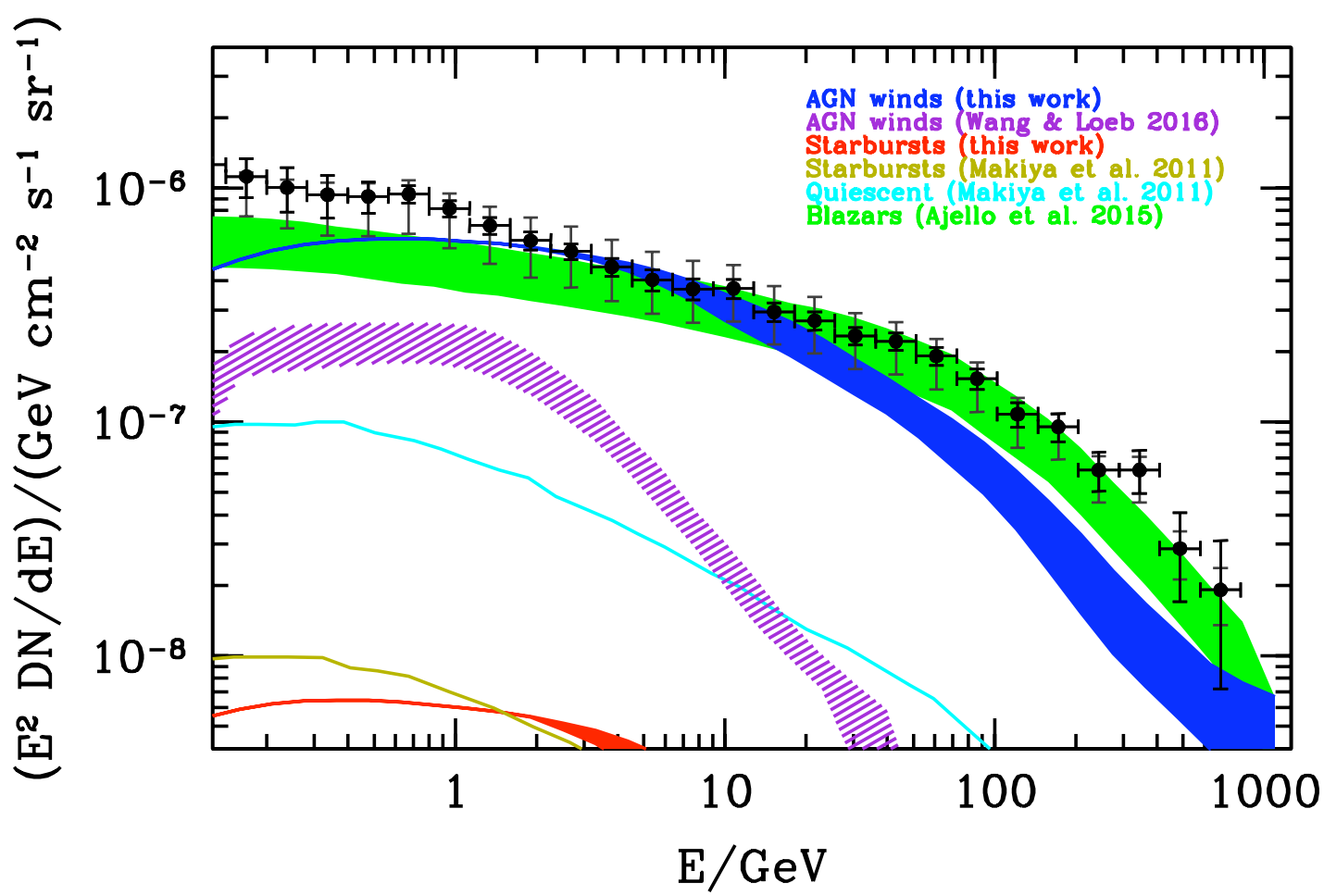

Fig. 6. EGB spectrum. The blue and red shaded regions correspond to AGN winds and starburst galaxies predicted by our SAM, respectively. The purple shaded region corresponds to the contribution to the EGB from AGN winds estimated by Wang \& Loeb (2016a). The cyan and gold lines indicate the estimate for quiescent and starburst galaxies, respectively, predicted by the Makiya et al. (2011) SAM. The green shaded region shows the contribution to the EGB from blazars as estimated by Ajello et al. (2015). The data points are as in Fig. 4.

interval $z=0-6$. Deriving the bolometric luminosity function from the observed luminosity functions in different electromagnetic bands is not a trivial procedure as it requires assumptions on the bolometric corrections, and corrections for obscured sources. Hopkins et al. (2007) use a luminosity-dependent bolometric correction, and they correct their data for extinction and the fraction of Compton-thick AGN missed in IR, optical, and $\mathrm{X}$-ray data. They also give analytical approximations of the empirical bolometric luminosity function. In their calculation, Wang \& Loeb (2016a) use the pure luminosity evolution (PLE) model given by Hopkins et al. (2007) to describe the evolution of the AGN population. As discussed by Hopkins et al. (2007), although the PLE model provides a reasonable lowest order of magnitude approximation to the data, it underpredicts the abundance of low-luminosity AGN at $z \lesssim 0.5$. Such an underprediction of faint objects explains the normalisation difference seen in Fig. 6.

As for the shape of the EGB spectrum, we find that while the two spectral shapes are consistent at energies $E_{\gamma} \lesssim 1 \mathrm{GeV}$, at higher energies the SAM predicts a decline in the EGB spectrum that begins at larger energies and is shallower than that predicted by Wang \& Loeb (2016a). The high-energy part of the predicted EGB spectrum is affected by $\gamma$-ray absorption in the intergalactic medium due to the EBL. There are large uncertainties regarding the EBL estimates and thus also the $\gamma$-ray optical depth used in Eq. (16) to calculate the EGB spectrum. We used $\tau_{\gamma \gamma}$ given by Stecker et al. (2016), while Wang \& Loeb (2016a) used the $\gamma$-ray optical depth estimated by the same authors in a previous paper (Stecker et al. 2007). The latter results in a larger absorption of $\gamma$-rays at energies $E_{\gamma} \gtrsim 10 \mathrm{GeV}$, and this could in part explain the discrepancy in the shape of the EGB spectra seen in Fig. 6. In fact, we have verified that assuming $\tau_{\gamma \gamma}$ given by
Stecker et al. (2007), the SAM predicts a steeper decline of the cumulative $\gamma$-ray emission from AGN winds, however the predicted EGB spectrum still remains shallower that that predicted by Wang \& Loeb (2016a).

It is also worth noting that the solutions of the hydrodynamical equations describing the outflow motion in an isothermal gas density profile give a constant outflow velocity (i.e. an energy-driven outflow) in the Lapi et al. (2005) model, and an outflow velocity that decreases with increasing outflow radius (i.e. a momentum-driven outflow) in the Wang \& Loeb (2016a) model. The shock velocity determines the maximum energy of accelerated protons, which in turn shapes the high-energy part of the EGB spectrum. The maximum energy that can attain accelerated protons can be expressed in terms of the shock velocity, size of the accelerator, and magnetic field strength as $E_{\max } \propto v_{\mathrm{s}} R_{\mathrm{S}} B$ (see Eq. (2)). Since $B_{\text {shock }} \propto R_{\mathrm{s}}^{-1}$ for an isothermal gas density profile, the maximum energy scales as $E_{\max } \propto v_{\mathrm{s}}$. Thus in an energy-driven outflow the proton maximum energy remains constant during the outflow expansion, while in a momentum-driven outflow $E_{\max }$ decreases with time.

As for the contribution to the EGB from star-forming galaxies, Makiya et al. (2011) derived the contribution from both starburst and quiescent galaxies using a SAM of hierarchical galaxy formation. As in our model, the star formation in starburst galaxies is triggered by galaxy interactions, while in quiescent galaxies the star formation is determined by the cold gas reservoir and the galaxy dynamical time-scale. In the Makiya et al. (2011) model the emission of quiescent and starburst galaxies are modelled based on templates which are tuned to reproduce the $\gamma$-ray spectra of the Milky Way and M82, respectively. For quiescent galaxies, they assumed the so-called escape regime. In this regime the energy losses of accelerated protons are dominated 


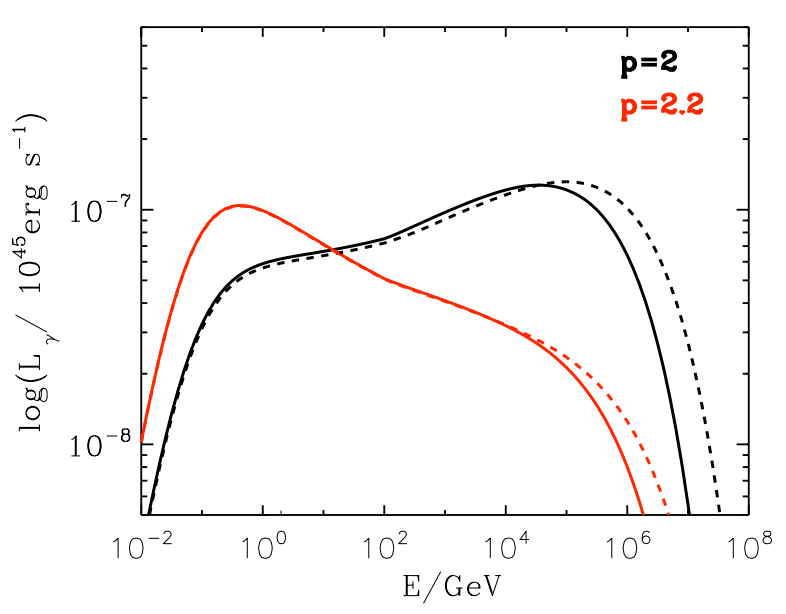

Fig. 7. Neutrino spectrum of an AGN with $L_{\mathrm{AGN}}=7 \times 10^{44} \mathrm{erg} / \mathrm{s}$ hosted in a halo of mass $10^{12} M_{\odot}$ at $z=0.1$. Model parameter as in Fig. 3 .

by their escape from the diffuse region of the galaxy, and the $\gamma$-ray luminosity depends on the SFR and mass of ISM gas, that is, $L_{\gamma} \propto S F R \times M_{\mathrm{c}}$. For the starburst galaxies, they assumed the calorimetric regime. In this case the $\gamma$-ray luminosity is no longer dependent on the gas mass, that is, $L_{\gamma} \propto S F R$. The authors determined the relation between $L_{\gamma}$ and $S F R \times M_{\mathrm{c}}$, and $L_{\gamma}$ and SFR, by fitting the results of four star-forming galaxies detected by Fermi-LAT. The contributions to the EGB from quiescent and starburst galaxies derived by Makiya et al. (2011) are shown in Fig. 6. They found that the contribution to the EGB from quiescent galaxies is a factor of $\sim 10$ larger than that provided by starburst galaxies. The latter agrees reasonably well with our estimate. This supports the scenario discussed in Sect. 5 where the $\gamma$-ray emission from star-forming galaxies (quiescent plus starburst) predicted by hierarchical galaxy formation models is lesser than that provided by AGN winds.

\subsection{Multi-messenger implications}

In Sect. 5 we show that a sizeable fraction of the EGB can be accounted by hadronic $\gamma$-ray emission from AGN winds. The hadronic origin of the $\gamma$-ray flux will make the production of neutrinos unavoidable, creating a diffuse neutrino background. Indeed, in proton-proton interactions approximately two thirds of the pions produced are charged pions that decay into muons and neutrinos followed by electrons and positrons and more neutrinos: $\pi^{+} \rightarrow \mu^{+}+v_{\mu}$ and $\mu^{+} \rightarrow \mathrm{e}^{+}+v_{\mathrm{e}}+\bar{v}_{\mu} ; \pi^{-} \rightarrow \mu^{-}+\bar{v}_{\mu}$ and $\mu^{-} \rightarrow \mathrm{e}^{-}+\bar{v}_{\mathrm{e}}+v_{\mu}$. Neutrinos can also be created in interactions of $\mathrm{CR}$ protons with the ambient radiation field. Here we focus on proton-proton interactions as they are the dominant process for AGN winds (Tamborra et al. 2014; Wang \& Loeb 2016b; Lamastra et al. 2016).

To calculate the energy spectra of neutrinos produced in AGN winds we use the parametrisations derived by Kelner et al. (2006; see Fig. 7). Then, the cumulative neutrino flux from AGN winds can be obtained by summing the neutrino emission over the entire AGN population, as described by Eq. (16). The absorption due to the EBL is ignored in the calculation of the neutrino background, and this introduces a dependence of the predicted spectrum on the values of the magnetic field in the shock region.

Figure 8 compares the cumulative neutrino background from AGN winds with the most recent IceCube data. The latter are fitted by two different models: a single power-law model, and a differential model with nine free parameters (Aartsen et al. 2015). We find that the estimated neutrino intensity is comparable, within the astrophysical uncertainties, to the IceCube measurement for spectral index $p \sim 2.2-2.3$. As shown in Fig. 5, these spectral indexes imply the largest contribution to the EGB from AGN winds at energies $E_{\gamma} \simeq 1-10 \mathrm{GeV}$, which, when added to the contribution from the balazar population, lead to a slight overestimate of Fermi-LAT data. Of course, any plausible model for the IceCube neutrino background should not overpopulate the gamma bounds. This tension points to a class of sources that are opaque in the $\gamma$-rays (Chakraborty \& Izaguirre 2016; Murase et al. 2016). As discussed in Chakraborty \& Izaguirre (2016), the large photon number density present in the environment of starburst galaxies implies that $\gamma$-rays could interact inside the galaxy before escape. The AGN-starburst connection predicted by our SAM implies that the internal absorption of $\gamma$-rays could also be present in AGN host galaxies. Thus, the Fermi-LAT and IceCube data could be reproduced simultaneously by our SAM (see also Murase et al. 2013; Wang \& Loeb 2016b).

The analysis of positional coincidence of IceCube neutrino events with known astrophysical sources is a difficult task owing to the poor angular resolution of the detector. For this reason, there are as yet no confirmed identifications for astrophysical sources of IceCube neutrino events (e.g. Aartsen et al. 2014; Adrián-Martínez et al. 2016b; The IceCube Collaboration et al. 2015). Recently, Padovani et al. (2016 see also Resconi et al. 2017) argued for a statistical significant correlation between IceCube neutrino events and high-energy peaked BL Lacertae (HBL) objects in the second catalogue of hard Fermi-LAT sources (Ackermann et al. 2016). Although HBL are promising neutrino-emitter candidates (Lucarelli et al. 2017), they can account for $\sim 10-20 \%$ of the IceCube signal.

In the near future, the better angular resolution of KM3NeT $\left(\sim 0.2^{\circ}\right.$ for neutrinos with energy $E \gtrsim 10 \mathrm{TeV}$, track-like events, Adrián-Martínez et al. 2016a) will allow us to effectively constrain the position of the possible counterparts of neutrino events, thus providing a possible direct test of neutrino background models.

\section{Conclusions}

We have incorporated the description of the hadronic $\gamma$-ray emission from relativistic protons accelerated in AGN-driven and $\mathrm{SN}$-driven shocks into a state-of-the-art SAM of hierarchical galaxy formation. Our SAM has already proven to match the statistical properties of the galaxy and AGN populations at low and high redshift and in different electromagnetic bands. We have compared the predictions for the cumulative $\gamma$-ray emission from AGN winds and star-forming galaxies with the latest measurement of the EGB performed by the Fermi-LAT in the range between $100 \mathrm{Mev}$ and $820 \mathrm{GeV}$ (Ackermann et al. 2015). The main results of this paper are as follows:

- In hierarchical clustering scenarios, connecting the physics of AGN and starburst galaxies to the merging histories of the host galaxies, assuming a comparable efficiency for accelerating protons in AGN-driven and SN-driven shocks $\left(\eta_{\mathrm{p}}=\right.$ 0.1 ), the contribution to the EGB from AGN winds dominates over that from starburst galaxies. If we consider also the contribution of the less powerful but more numerous quiescent galaxies, the contribution to the EGB from all starforming galaxies is a factor $\sim 3-5$ lower than that provided by AGN winds. 


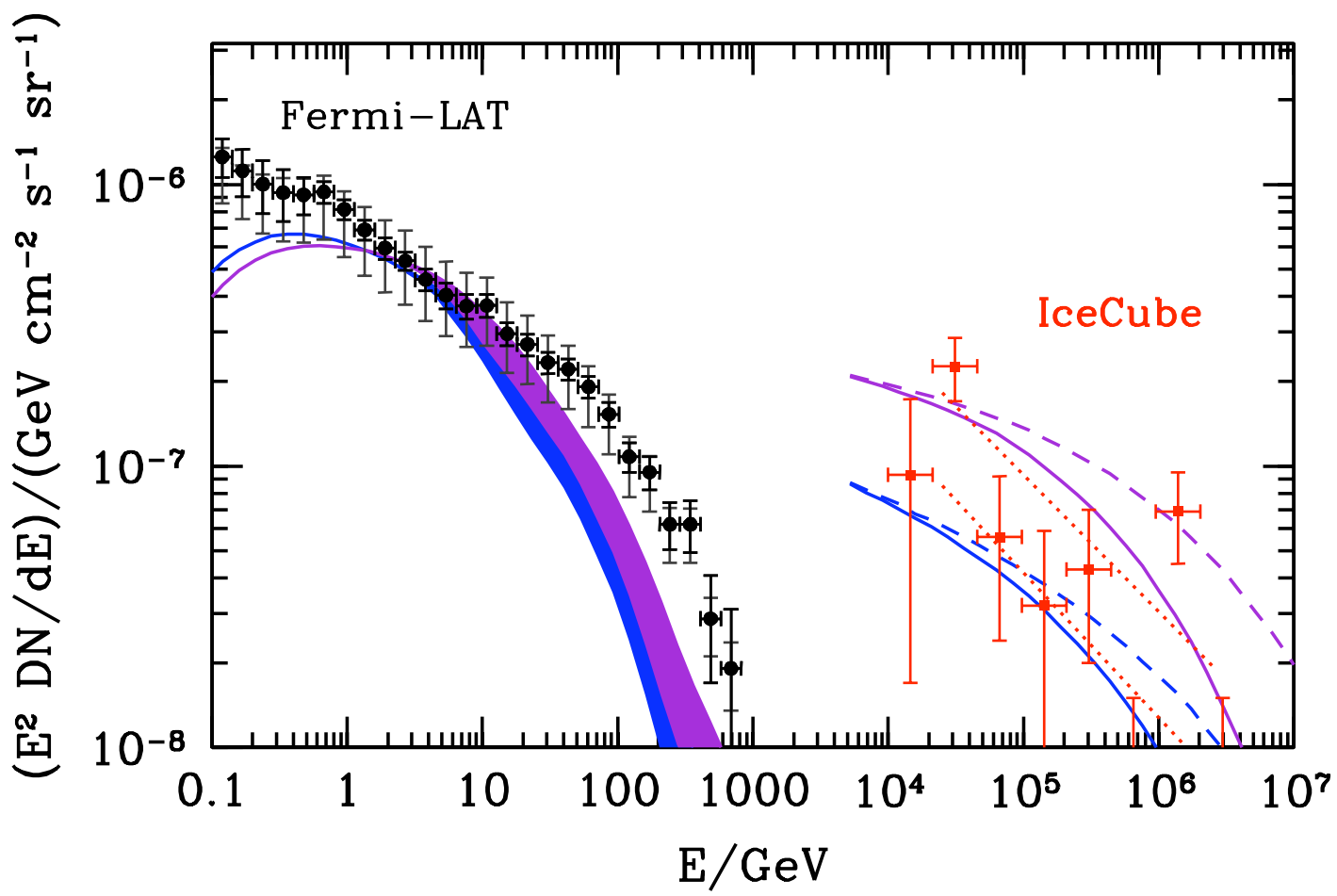

Fig. 8. Cumulative $\gamma$-ray (left) and neutrino (right) background from AGN winds predicted by our SAM. Model predictions are shown for our fiducial model and for different AGN wind spectral energy parameters: $p=2.2$ (purple lines), $p=2.3$ (blue lines), $B=B_{\mathrm{ISM}}$ (solid lines), and $B=B_{\text {shock }}$ dashed lines. The circles represent Fermi-LAT data (Ackermann et al. 2015). The squares show the differential model of IceCube neutrino data, while purple dotted lines represent the power-law models (Aartsen et al. 2015).

- The cumulative $\gamma$-ray emission from AGN winds and blazars can account for the amplitude and spectral shape of the EGB, assuming the standard acceleration theory, and AGN wind parameters that agree with observations. At energies lower and greater than $E_{\gamma} \simeq 10 \mathrm{GeV}$ the EGB is dominated by AGN winds and blazars, respectively. The transition between these two regimes could, in principle, give rise to breaks and features in the EGB energy spectrum.

- The neutrino background resulting from charged pion decays following hadronic interactions can reproduce the IceCube data assuming accelerated proton spectral index $p \sim 2.2-2.3$. The Fermi-LAT data could be reproduced simultaneously, taking into account internal absorption of $\gamma$-rays.

\section{References}

Aartsen, M. G., Ackermann, M., Adams, J., et al. 2014, ApJ, 796, 109 Aartsen, M. G., Abraham, K., Ackermann, M., et al. 2015, ApJ, 809, 98 Ackermann, M., Ajello, M., Allafort, A., et al. 2012, ApJ, 755, 164 Ackermann, M., Ajello, M., Albert, A., et al. 2015, ApJ, 799, 86 Ackermann, M., Ajello, M., Atwood, W. B., et al. 2016, ApJS, 222, 5

Ackermann, M., Ajello, M., Albert, A., et al. 2017, ApJ, 836, 208

Adrián-Martínez, S., Ageron, M., Aharonian, F., et al. 2016a, J. Phys. G Nucl. Phys., 43, 084001

Adrián-Martínez, S., Albert, A., André, M., et al. 2016b, ApJ, 823, 65 Aharonian, F. A., \& Atoyan, A. M. 2000, A\&A, 362, 937

Ajello, M., Gasparrini, D., Sánchez-Conde, M., et al. 2015, ApJ, 800, L27 Antonucci, R. R. J., \& Miller, J. S. 1985, ApJ, 297, 621

Atwood, W. B., Abdo, A. A., Ackermann, M., et al. 2009, ApJ, 697, 1071

Bell, A. R. 1978a, MNRAS, 182, 147

Bell, A. R. 1978b, MNRAS, 182, 443

Blandford, R. D., \& Ostriker, J. P. 1978, ApJ, 221, L29

Bond, J. R., Cole, S., Efstathiou, G., \& Kaiser, N. 1991, ApJ, 379, 440

Bruzual, G., \& Charlot, S. 2003, MNRAS, 344, 1000

Calura, F., \& Menci, N. 2009, MNRAS, 400, 1347
Cavaliere, A., \& Vittorini, V. 2000, ApJ, 543, 599

Chakraborty, N., \& Fields, B. D. 2013, ApJ, 773, 104

Chakraborty, S., \& Izaguirre, I. 2016, ArXiv e-prints [arXiv: 1607.03361]

Chartas, G., Brandt, W. N., Gallagher, S. C., \& Garmire, G. P. 2002, ApJ, 579 , 169

Chevalier, R. A. 1976, ApJ, 207, 872

Chevalier, R. A. 1982, ApJ, 258, 790

Chevalier, R. A. 1998, ApJ, 499, 810

Cicone, C., Maiolino, R., Sturm, E., et al. 2014, A\&A, 562, A21

Di Mauro, M., Calore, F., Donato, F., Ajello, M., \& Latronico, L. 2014, ApJ, 780,161

Drury, L. 1983, Space Sci. Rev., 36, 57

Faucher-Giguère, C.-A., \& Quataert, E. 2012, MNRAS, 425, 605

Ferrara, A., Pettini, M., \& Shchekinov, Y. 2000, MNRAS, 319, 539

Feruglio, C., Maiolino, R., Piconcelli, E., et al. 2010, A\&A, 518, L155

Feruglio, C., Fiore, F., Carniani, S., et al. 2015, A\&A, 583, A99

Fields, B. D., Pavlidou, V., \& Prodanović, T. 2010, ApJ, 722, L199

Fiore, F., Feruglio, C., Shankar, F., et al. 2017, A\&A 601, A143

Fornasa, M., \& Sánchez-Conde, M. A. 2015, Phys. Rep., 598, 1

Franco, J., Tenorio-Tagle, G., Bodenheimer, P., \& Rozyczka, M. 1991, PASP, 103,803

García-Burillo, S., Combes, F., Usero, A., et al. 2014, A\&A, 567, A125

Gatti, M., Lamastra, A., Menci, N., Bongiorno, A., \& Fiore, F. 2015, A\&A, 576, A32

Giallongo, E., Menci, N., Fiore, F., et al. 2012, ApJ, 755, 124

Harrison, C. M., Alexander, D. M., Mullaney, J. R., et al. 2016, MNRAS, 456, 1195

Hopkins, P. F., Richards, G. T., \& Hernquist, L. 2007, ApJ, 654, 731

Inoue, Y. 2011, ApJ, 733, 66

Kauffmann, G. 1996, MNRAS, 281, 475

Kauffmann, G., \& Charlot, S. 1998, MNRAS, 294, 705

Kelner, S. R., Aharonian, F. A., \& Bugayov, V. V. 2006, Phys. Rev. D, 74, 034018

Keshet, U., Waxman, E., Loeb, A., Springel, V., \& Hernquist, L. 2003, ApJ, 585, 128

King, A. 2003, ApJ, 596, L27

King, A. R., \& Pounds, K. A. 2003, MNRAS, 345, 657

King, A., \& Pounds, K. 2015, ARA\&A, 53, 115

King, A. R., Zubovas, K., \& Power, C. 2011, MNRAS, 415, L6

Lacey, C., \& Cole, S. 1993, MNRAS, 262, 627 
Lacki, B. C., Thompson, T. A., \& Quataert, E. 2010, ApJ, 717, 1

Lacki, B. C., Horiuchi, S., \& Beacom, J. F. 2014, ApJ, 786, 40

Lamastra, A., Menci, N., Maiolino, R., Fiore, F., \& Merloni, A. 2010, MNRAS, 405, 29

Lamastra, A., Menci, N., Fiore, F., \& Santini, P. 2013a, A\&A, 552, A44

Lamastra, A., Menci, N., Fiore, F., et al. 2013b, A\&A, 559, A56

Lamastra, A., Fiore, F., Guetta, D., et al. 2016, A\&A, 596, A68

Lapi, A., Cavaliere, A., \& Menci, N. 2005, ApJ, 619, 60

Lucarelli, F., Pittori, C., Verrecchia, F., et al. 2017, ApJ, 846, 121

Madau, P., \& Rees, M. J. 2001, ApJ, 551, L27

Makiya, R., Totani, T., \& Kobayashi, M. A. R. 2011, ApJ, 728, 158

Marconi, A., Risaliti, G., Gilli, R., et al. 2004, MNRAS, 351, 169

McBride, J., Quataert, E., Heiles, C., \& Bauermeister, A. 2014, ApJ, 780, 182

Menci, N., Cavaliere, A., Fontana, A., Giallongo, E., \& Poli, F. 2002, ApJ, 575, 18

Menci, N., Fontana, A., Giallongo, E., \& Salimbeni, S. 2005, ApJ, 632, 49

Menci, N., Fontana, A., Giallongo, E., Grazian, A., \& Salimbeni, S. 2006, ApJ, 647,753

Menci, N., Fiore, F., Puccetti, S., \& Cavaliere, A. 2008, ApJ, 686, 219

Menci, N., Gatti, M., Fiore, F., \& Lamastra, A. 2014, A\&A, 569, A37

Mo, H. J., Mao, S., \& White, S. D. M. 1998, MNRAS, 295, 319

Murase, K., Ahlers, M., \& Lacki, B. C. 2013, Phys. Rev. D, 88, 121301

Murase, K., Guetta, D., \& Ahlers, M. 2016, Phys. Rev. Lett., 116, 071101

Narayan, C. A., \& Jog, C. J. 2002, A\&A, 394, 89

Nims, J., Quataert, E., \& Faucher-Giguère, C.-A. 2015, MNRAS, 447, 3612

Ostriker, J. P., \& McKee, C. F. 1988, Rev. Mod. Phys., 60, 1

Padovani, P., Resconi, E., Giommi, P., Arsioli, B., \& Chang, Y. L. 2016, MNRAS, 457, 3582

Pounds, K. A., Reeves, J. N., King, A. R., et al. 2003, MNRAS, 345, 705

Reeves, J. N., O’Brien, P. T., \& Ward, M. J. 2003, ApJ, 593, L65

Resconi, E., Coenders, S., Padovani, P., Giommi, P., \& Caccianiga, L. 2017, MNRAS, 468, 597

Reynolds, S. P. 2008, ARA\&A, 46, 89
Robishaw, T., Quataert, E., \& Heiles, C. 2008, ApJ, 680, 981

Rupke, D. S. N., \& Veilleux, S. 2011, ApJ, 729, L27

Sanders, D. B., \& Mirabel, I. F. 1996, ARA\&A, 34, 749

Shen, Y. 2016, ApJ, 817, 55

Stecker, F. W. 1970, Ap\&SS, 6, 377

Stecker, F. W., \& Venters, T. M. 2011, ApJ, 736, 40

Stecker, F. W., Malkan, M. A., \& Scully, S. T. 2007, ApJ, 658, 1392

Stecker, F. W., Scully, S. T., \& Malkan, M. A. 2016, ApJ, 827, 6

Tamborra, I., Ando, S., \& Murase, K. 2014, J. Cosmol. Astropart. Phys., 9, 043

Tatischeff, V. 2008, ArXiv eprint [arXiv: 0804 . 1004]

The IceCube Collaboration, Aartsen, M. G., Abraham, K., et al. 2015, ArXiv eprint [arXiv: 1510.05222]

Thompson, T. A., Quataert, E., Waxman, E., Murray, N., \& Martin, C. L. 2006, ApJ, 645, 186

Tombesi, F., Sambruna, R. M., Reeves, J. N., et al. 2010, ApJ, 719, 700

Tombesi, F., Cappi, M., Reeves, J. N., et al. 2013, MNRAS, 430, 1102

Tombesi, F., Meléndez, M., Veilleux, S., et al. 2015, Nature, 519, 436

van der Kruit, P. C., \& Freeman, K. C. 2011, ARA\&A, 49, 301

van Weeren, R. J., Brunetti, G., Brüggen, M., et al. 2016, ApJ, 818, 204

Vazza, F., Eckert, D., Brüggen, M., \& Huber, B. 2015, MNRAS, 451, 2198

Vazza, F., Brüggen, M., Wittor, D., et al. 2016, MNRAS, 459, 70

Wang, X., \& Fields, B. D. 2016, ApJ, submitted [arXiv: 1612 . 07290]

Wang, X., \& Loeb, A. 2015, MNRAS, 453, 837

Wang, X., \& Loeb, A. 2016a, Nat. Phys., 12, 1116

Wang, X., \& Loeb, A. 2016b, J. Cosmol. Astropart. Phys., 12, 012

Weaver, R., McCray, R., Castor, J., Shapiro, P., \& Moore, R. 1977, ApJ, 218, 377

Yoast-Hull, T. M., Gallagher, III, J. S., Zweibel, E. G., \& Everett, J. E. 2014, ApJ, 780, 137

Yu, Q., \& Tremaine, S. 2002, MNRAS, 335, 965

Zakamska, N. L., Hamann, F., Pâris, I., et al. 2016, MNRAS, 459, 3144

Zubovas, K., \& King, A. 2012, ApJ, 745, L34

Zubovas, K., \& Nayakshin, S. 2014, MNRAS, 440, 2625 